\title{
Effects of fabrication technique upon material properties and permeation characteristics of palladium-gold alloy membranes for hydrogen separations
}

\author{
Sabina K. Gade ${ }^{1}$, Kent E. Coulter ${ }^{2}$ and J. Douglas Way ${ }^{1 *}$ \\ www.goldbulletin.org
}

\begin{abstract}
This work highlights new research into the fundamental properties of palladium-gold alloy membranes. Two types of self-supported palladium-gold foils were studied; membranes produced by magnetron sputtering and membranes produced by cold-working. The cold-worked membranes had thicknesses of 25 microns and gold contents from 0-40 wt\% $\mathrm{Au}$, while the sputtered films ranged from 10-31 microns in thickness and 5-10 wt\% Au. These films were characterized by single-gas permeation testing in the temperature range of $473-773 \mathrm{~K}$ and at pressures of up to $772 \mathrm{kPa}$. Membranes were studied before and after testing by XRD, XPS, XRF, and SEM/EDS.
\end{abstract}

Hydrogen permeability in the $0-20$ wt Au\% range was found to be a function of synthesis technique as much as alloy content, with no single alloy having superior permeability at all temperatures. Sputtered materials had generally higher permeability than cold-worked materials of equivalent composition, although the thicker sputtered membrane had reduced hydrogen permeability compared to its thinner counterparts.

\footnotetext{
Colorado School of Mines, Department of Chemical Engineering, Golden, CO, 80401, U.S.A.;

Southwest Research Institute, 6220 Culebra Rd., San Antonio, TX, 78238-5166
}

Tel: 001303273 3519, Fax: 0013032733730

E-mail:sgade@mines.edu, kent.coulter@swri.com

* Corresponding author
E-mail: dway@mines.edu

Keywords: palladium gold membrane, palladium alloy segregation, ordered surface phase, magnetron sputtering, cold working, oxidation history
In this composition range, the addition of gold generally acted to reduce activation energy of hydrogen permeation. The differences in membrane permeability by fabrication technique are primarily attributed to preferential orientation effects. These effects also appear to contribute to other permeation phenomena, such as low-temperature hydrogen embrittlement, the dependence of flux on feed pressure, and the formation of long range ordered surface phases.

\section{Introduction}

The desire to reduce carbon dioxide $\left(\mathrm{CO}_{2}\right)$ emissions and improve energy efficiency has made hydrogen $\left(\mathrm{H}_{2}\right)$ an increasingly appealing fuel source. PEM fuel cells, in particular, require a highly pure hydrogen feedstock, free from sulphur or carbon monoxide, to avoid poisoning their sensitive lowtemperature catalysts [1]. As most hydrogen is currently produced by reforming of hydrocarbon sources, particularly natural gas, significant new research has been conducted into economical ways to produce high purity hydrogen from these mixedgas streams [2]. Presently, the dominant method of hydrogen purification is pressure swing adsorption (PSA). While PSA is a mature and well-established technology, adsorbent loading is reduced at the high temperatures typical of $\mathrm{H}_{2}$ production, it is costly due to the required low purge pressures, and the requirement that the non-hydrogen stream components extracted are at low pressures and require compression for $\mathrm{CO}_{2}$ sequestration processes [3].

One alternative method to PSA is the use of dense, metallic membranes. Palladium (Pd), platinum (Pt), nickel $(\mathrm{Ni})$, and the metallic elements in group III-V of the periodic table all have the ability to selectively permeate hydrogen through a solution- 
diffusion mechanism, in which molecular hydrogen dissociatively adsorbs onto the high-pressure feed surface, is transported through the membrane via a site-hopping mechanism, then recombines and desorbs on the low-pressure permeate side (Figure 1) [4]. Because only hydrogen can be transported in this fashion, dense, pinhole-free films of these metals can produce hydrogen that, in theory, will be infinitely pure. Palladium is of particular interest for this purpose, as it has high catalytic activity for hydrogen dissociation combined with high permeability of the dissolved hydrogen [5]. However, in practice, commercial application of palladium has been limited to small-scale, ultra high purity applications. This is due to several issues: pure palladium tends to swell and crack when exposed to hydrogen at temperatures below 573K, carbon can contaminate the surface and depress permeability, and sulphur compounds can permanently poison the material.

In order to obviate these issues, as well as improve permeability, palladium is frequently alloyed with a wide range of other metals. The choice of the alloying element or elements can be tailored to the application in question: as an example, alloys with silver have high hydrogen permeability and resistance to embrittlement, and are commercially used for lowtemperature upgrading of industrial hydrogen to ultra high purity [6]. For high-temperature cleanup of dirty gases, such as those coming off a methane watergas shift reactor, different materials are required. As early as 1967, it was demonstrated that alloys of palladium with 1-20 wt\% gold had up to $30 \%$ higher

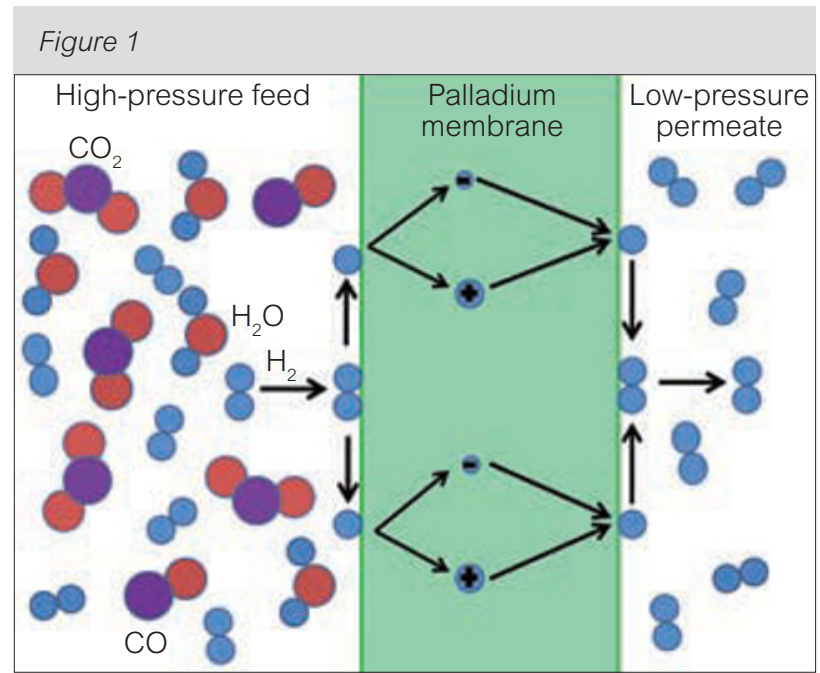

Solution-diffusion transport of hydrogen through dense palladium membrane hydrogen permeability than pure palladium, and that membranes containing 40 wt \% gold suffered almost no flux inhibition when used in streams containing 4 ppm hydrogen sulphide (Figure 2) [7, 8]. By contrast, although the most heavily commercialized alloy, $\mathrm{Pd}_{77} \mathrm{Ag}_{23}$, has four times the permeability of $\mathrm{Pd}_{60} \mathrm{Au}_{40}$ under pure gas conditions, under 4 ppm sulphur the silver alloy has only $1 / 20^{\text {th }}$ of the gold alloy's permeability. Similar flux inhibition by $\mathrm{H}_{2} \mathrm{~S}$ is observed for other commercial alloys such as $\mathrm{Pd}_{60} \mathrm{Cu}_{40}$, while highly permeable Pd-Y alloys operate most successfully at low temperatures which will promote sulphide formation [9]. Since sulphur is a common contaminant in hydrocarbon streams from both renewable and fossil sources, this makes the Pd-Au system particularly appealing for real-world purification applications. In previous work, we have successfully produced palladiumgold alloys by sequential electroless plating, creating both supported composite membranes and freestanding foils $[10,11]$. The plated materials have the same stability and tolerance to typical reformate streams as cold rolled materials, but due to the method of fabrication, tend to have enhanced surface gold contents and may not be entirely similar to homogeneous alloys [12]. The role of this work, therefore, is to improve understanding of the fundamental properties of the Pd-Au system, using homogeneous materials. Freestanding foils were chosen to eliminate any support effects.

\section{Materials and methods}

Two types of self-supported membranes were examined: Sputtered films, produced by Southwest Research Institute ${ }^{\circledR}\left(S w R I^{\circledR}\right)$, and cold-worked films, produced by Tanaka Kikinzoku Kogya International (TKK) and Alfa Aesar (Alfa). The sputtered films were deposited onto smooth, thermally oxidized 6"silicon wafers. A 5"X12"Pd sputter target with Au foil tiles placed on the surface of the target was utilized to control Pd-Au composition. The sputter target was run at 780 watts which resulted in a deposition rate of $\sim 0.6 \mathrm{~nm} /$ second. The silicon wafer is rotated above the target at a distance of 6" to generate a uniform film over the surface of the wafer. Upon completion of the coating process the coated wafers were stored for one day at ambient temperature and atmospheric pressure and then scored at the edge of the wafer. The coating peeled off to form a freestanding film which was annealed at $673 \mathrm{~K}$ in argon for 2 hours.

The cold worked films and sputtered films were tested in their as-received conditions, without any 


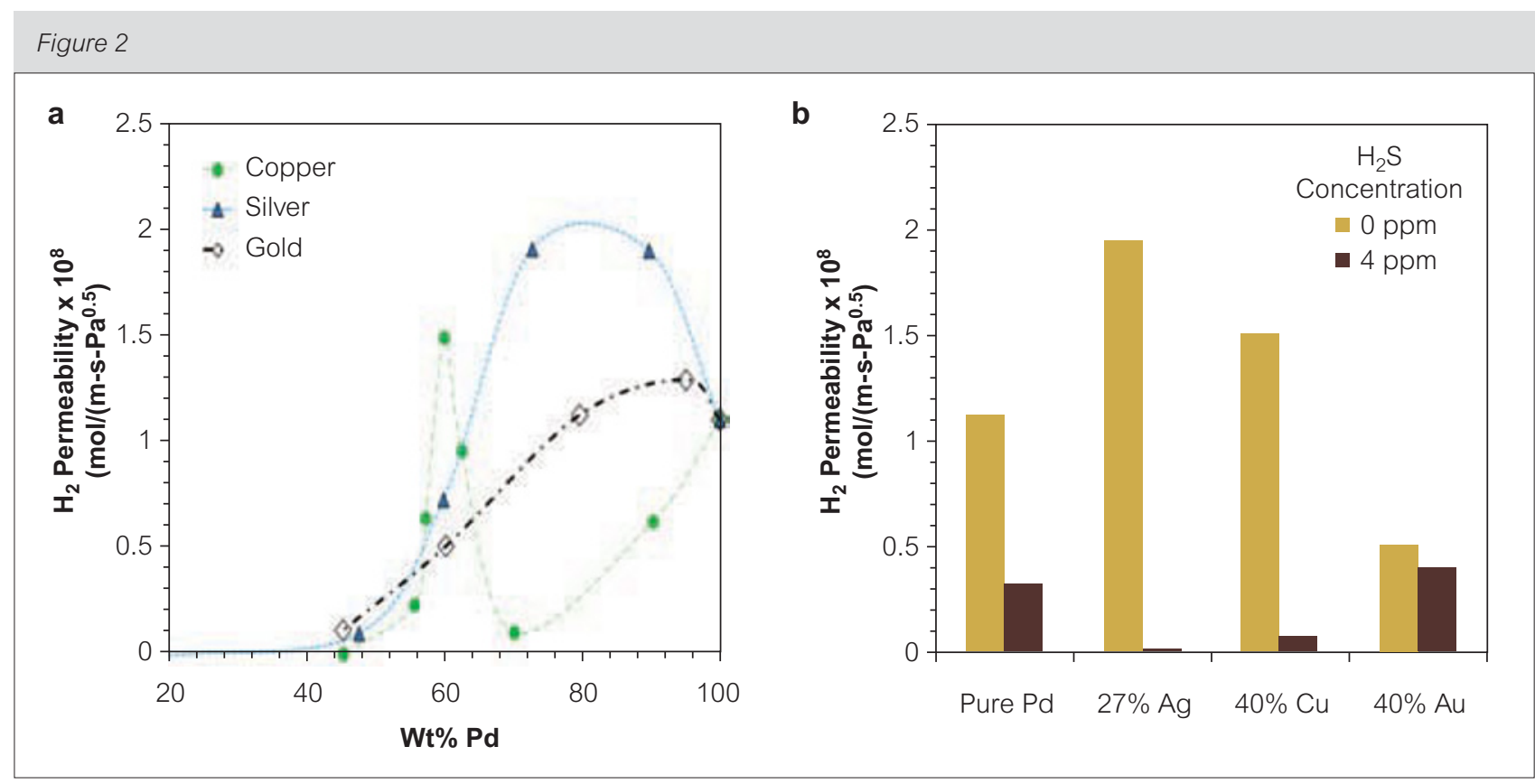

Hydrogen permeability at $623 \mathrm{~K}$ for various alloys of palladium as a function of a) Alloy composition and b) alloy and presence of $\mathrm{H}_{2} \mathrm{~S}$ in feed

preconditioning or pre-annealing apart from that conducted by the manufacturers. Foils were mounted in modified Millipore stainless steel permeation cells with graphite gaskets. The membranes were heated to $573 \mathrm{~K}$ at $60 \mathrm{~K} / \mathrm{h}$ under flowing air, which combined the heating and air oxidation steps of permeation testing into a single process. Single-gas permeation experiments with industrial-grade (99.9+\% purity) $\mathrm{H}_{2}$ and $\mathrm{N}_{2}$ were performed at pressure gradients up to $689.5 \mathrm{kPa}$, with permeate flows measured by a soap film meter. Temperature was stepped up in $50 \mathrm{~K}$ increments until $773 \mathrm{~K}$, then stepped down in $50 \mathrm{~K}$ increments until the membrane reached $473 \mathrm{~K}$ or developed a detectable leak. The permeate side was kept at local atmospheric pressure, approximately $82 \mathrm{kPa}$ in Golden, Colorado.

After testing was completed, membranes were cooled under flowing $\mathrm{N}_{2}$. Four characterizations were performed, each sampling at a different depth into the membrane: XPS (x-ray photoelectron spectroscopy), SEM/EDS (scanning electron microscopy/energy dispersive $x$-ray spectroscopy), XRD (x-ray diffraction), and XRF (x-ray fluorescence spectroscopy). These experiments were performed both before and after testing to observe how permeation experiments changed the materials. The XPS instrument was a Kratos Analytical HSI, using an $\mathrm{Al}-\mathrm{K} \alpha$ incident $\mathrm{x}$-ray source, with a chamber pressure between $5 \times 10^{-9}$ and $5 \times 10^{-10}$ torr. The XPS instrument samples only the top $8 \mathrm{~nm}$ of the surface. SEM/EDS was performed in an environmental scanning electron microscope, which penetrates the top 1-2 microns of the surface. The $x$-ray diffractometer used $\mathrm{Cu}-\mathrm{K} \alpha$ incident $\mathrm{x}$-radiation $(\lambda=1.54056 \AA)$, a step size of $0.01^{\circ}$, and a count time of 2 seconds per degree. For alloys of gold and palladium, $99.9 \%$ of the diffracted signal originates from the top 6 microns of the film. Glancing-incidence XRD was also performed, in which the incident angle was fixed at $4^{\circ}$, across the same range of $2 \theta$ as in standard XRD. This allows the XRD to focus on the top 0.6-0.8 microns of the film. Lastly, XRF was used to examine bulk thickness and composition. A Fischerscope ${ }^{\circledR}$ X-Ray $\mathrm{XLDM}^{\circledR}$-C4 device was used with a $50 \mathrm{kV}$ accelerating voltage and a tungsten filament.

\section{Permeation testing}

Six cold-worked membranes were studied: a pure Pd membrane (Alfa) and 5, 10, 15, 20, and $40 \mathrm{wt} \%$ Au alloys (TKK). All cold-worked foils had nominal thicknesses of 25.4 microns. Compositions as measured by EDS were close to and within predicted instrumental error ranges of the nominal compositions (Table 1), as were thicknesses when measured with a micrometer. In addition, three membranes were fabricated at SwRI using magnetron sputtering. Two membranes had nominal thicknesses of 10 
Table 1: Membrane thicknesses as measured by gravimetric analysis and compositions as measured by EDSIXRF

\begin{tabular}{|c|c|c|c|c|c|}
\hline Membrane & $\begin{array}{r}\text { Nominal } \\
\text { composition } \\
(w t \% A u)\end{array}$ & $\begin{array}{r}\text { Thickness } \\
(\mu \mathrm{m})\end{array}$ & $\begin{array}{r}\text { Side A } \\
(w t \% \text { Au, EDS })\end{array}$ & $\begin{array}{r}\text { Side B } \\
(w t \% \text { Au, EDS })\end{array}$ & $\begin{array}{r}\text { Overall } \\
(w t \% \text { Au, XRF) }\end{array}$ \\
\hline \multicolumn{6}{|c|}{ Cold-worked } \\
\hline Alfa Pure Pd & 0 & 25 & 0 & 0 & 0 \\
\hline TKK 5\% Au & 5 & 25 & 5.0 & 5.3 & 5.5 \\
\hline TKK 10\% Au & 10 & 25 & 11.5 & 11.4 & 10.2 \\
\hline TKK 15\% Au & 15 & 24 & 13.4 & 14.6 & 14.9 \\
\hline TKK 20\% Au & 20 & 25 & 20.6 & 21.3 & 19.0 \\
\hline TKK 40\% Au & 40 & 25 & 37.5 & 41.1 & 38.5 \\
\hline \multicolumn{6}{|c|}{ Sputtered } \\
\hline SWRI 44 & 5 & 8 & 8.8 & 7.5 & 7.0 \\
\hline SWRI 46 & 10 & 12 & 8.3 & 10.2 & 9.2 \\
\hline SWRI 126 & 10 & 31 & 13.2 & 8.9 & 10.1 \\
\hline
\end{tabular}

Figure 3

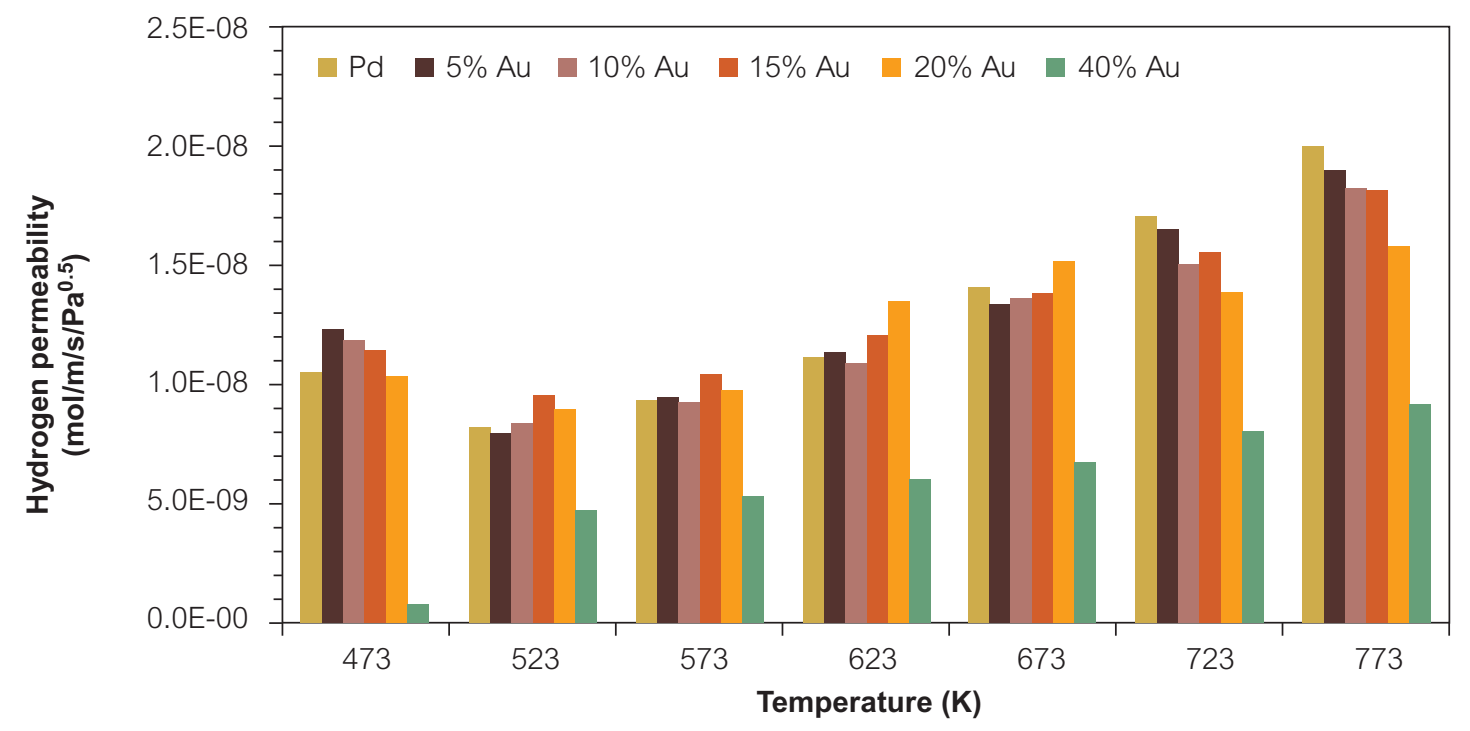

Hydrogen permeability for cold-worked foils as a function of temperature

microns while the third had a nominal thickness of 25 microns, and the compositions were nominally 5 and $10 \mathrm{wt} \%$ gold. The sputtering process is more difficult to control than cold-working, and membranes had different compositions across their thicknesses, though measuring different spots on the same side of a given membrane produced relatively consistent results.

All cold-worked membranes were stable and had zero detectable nitrogen permeability (i.e. no flow through pinholes or seals) for temperatures of $473 \mathrm{~K}$ or greater over the range of pressures tested. No single composition had consistently higher hydrogen permeability than the others, with different alloys being most permeable at different temperatures (figure 3). An unexpected phenomenon was noted, in that at $473 \mathrm{~K}$, the permeability for all alloys except the $40 \mathrm{wt} \%$ Au was significantly larger than that of the same alloy at higher temperatures. The membrane hydrogen permeabilities also displayed a linear relationship to the pressure gradient 


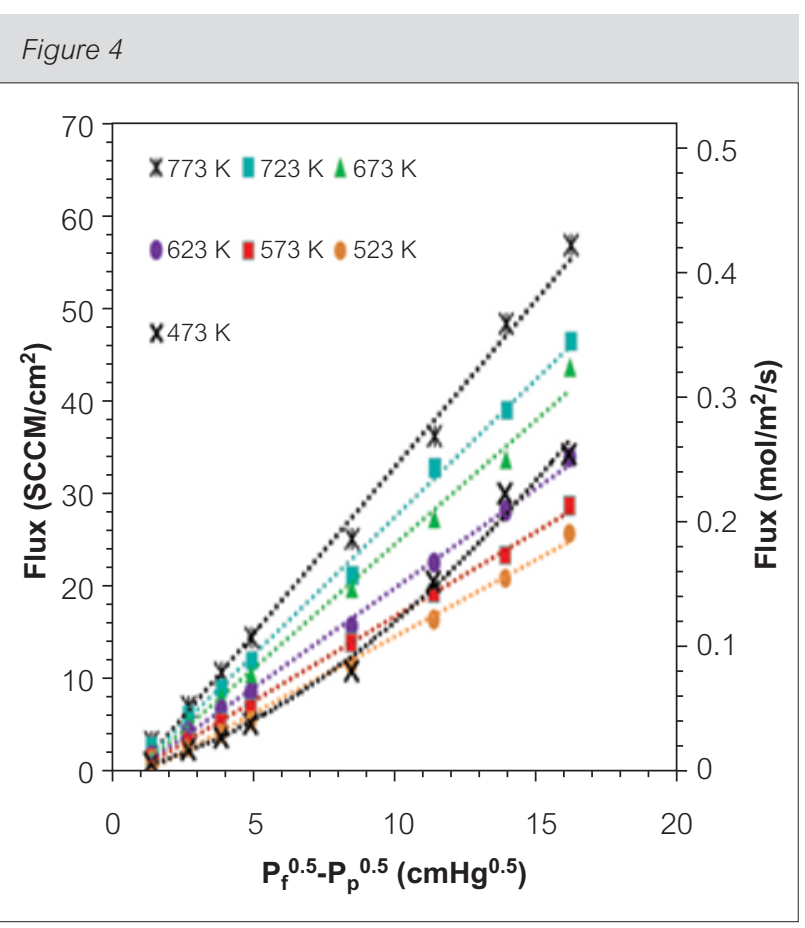

Hydrogen flux as a function of the square root of pressure gradient for a $25 \mu \mathrm{m}, 10 \mathrm{wt} \%$ Au cold-worked foil at temperatures from $472-773 \mathrm{~K}$

$\left(\sqrt{ } P_{\text {feed }}-\sqrt{ } P_{\text {permeate }}\right)$ above $523 \mathrm{~K}$, but had a squared dependence on this pressure gradient at lower temperatures, being linearly related to $\Delta \mathrm{P}$ (Figure 4). The "n-values", or pressure exponents, relating flux to thickness at temperatures above $523 \mathrm{~K}$ are consistently in the range of $0.5-0.75$, suggesting at these temperatures, the rate-limiting step in hydrogen transport is diffusion through the bulk. This is similar to the literature for pure palladium membranes $>1$ micron in thickness and temperatures greater than $573 \mathrm{~K}$ [13]. At temperatures of $573 \mathrm{~K}$ and lower, the $n$-values increase, implying that some surface process is becoming proportionally more important.

The most plausible explanation for both these phenomena is that the membranes were becoming slightly hydrogen embrittled, forming a $\beta$-hydride phase with a larger lattice parameter than the Pd-Au metallic lattice. The presence of the hydride phase is known to affect surface processes, producing a system with different activation energies at higher and lower temperatures $[14,15]$. While the hydride phase that forms at $473 \mathrm{~K}$ did affect permeability, it did not appear to damage the membranes: they could be cycled back to higher temperatures without showing any hysteresis effects of hydrogen permeability, and could be kept at $473 \mathrm{~K}$ for up to 72 hours without developing detectable nitrogen permeability. However, the fact that the membrane was leak-free at these conditions should not be taken as a statement of the relative low-T stability of the PdAu system, but as a function of the thickness of the membrane. At 423K, where embrittlement was more extensive, the membranes failed within three hours of exposure to hydrogen at $220 \mathrm{kPa}$ feed pressure, and temperature cycling showed significant permeability hysteresis. Thinner films of similar materials could be expected to undergo destructive embrittlement at higher temperatures.

The SwRI membranes were tested under similar operating conditions, although feed pressure was restricted to no greater than $633 \mathrm{kPa}$ to avoid placing excessive mechanical stress on the two thinner films. Unlike the thicker cold-worked materials, both SwRI-44 and SwRI-46 membranes developed trace amounts of nitrogen permeability at $473 \mathrm{~K}$, after being leak-free at all other temperatures tested. SwRI126 ruptured catastrophically at $473 \mathrm{~K}$ and $633 \mathrm{kPa}$ pressure, unlike any other self-supporting $\mathrm{Pd}-\mathrm{Au}$ foil studied in this work including the thinner coldworked membranes. Despite evidence of hydrogen embrittlement at low temperatures, the increase in $\mathrm{n}$-value at $473 \mathrm{~K}$ is much smaller for these films, with a square root dependence on pressure gradient still fitting the data appropriately. In addition to this, the SwRI membranes exhibited a significant correlation between thickness and permeability, despite their

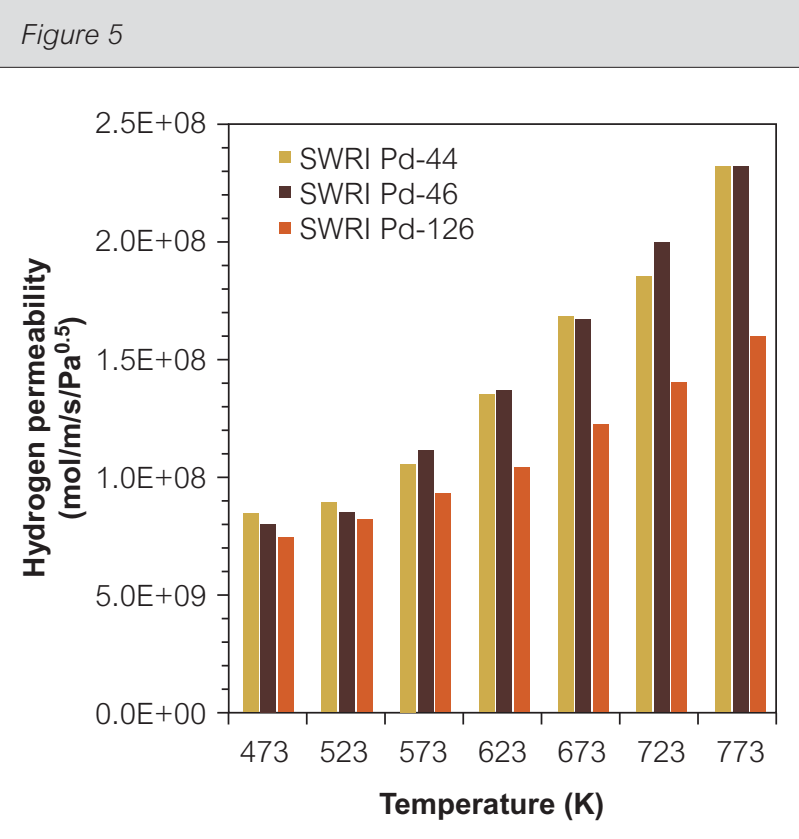

Hydrogen permeability as a function of temperature for sputtered foils 
similar compositions, with the thick membrane SwRI-126 having much lower permeability than the two thinner foils (Figure 5). Permeability being dependent on thickness suggests a surface process may be relevant.

All membrane permeabilities were fit with Arrhenius relationships in a temperature range from $523-773 \mathrm{~K}$. These results are summarized in Table 2. Multiple factors appear to affect hydrogen permeation behavior. Between 0-20 wt\% Au a general reduction of activation energy can be observed as gold is added, reducing the permeability temperature dependence. A simultaneous decrease in preexponential factor with increased gold content maintains all permeabilities in the same approximate range. Between 20 and $40 \mathrm{wt} \% \mathrm{Au}$, the activation energy begins to increase, suggesting that a different rate-limiting process may be beginning to dominate as the gold content rises.

Table 2: Activation energies and pre-exponential factors for Arrhenius fits of hydrogen permeability and temperature. The fit is restricted to temperatures above $473 \mathrm{~K}$ and a $95 \%$ confidence interval is given

\begin{tabular}{|lrr|}
\hline Membrane & $\begin{array}{r}\mathbf{P}_{\mathbf{0}} \times \mathbf{1 0}^{\mathbf{8}} \\
\text { (mol/m/s/Pa }\end{array}$ & Ea $\mathbf{( J / m o l )}$ \\
\hline \multicolumn{3}{|c|}{ Cold-worked } \\
\hline Pure Pd & $13.5 \pm 3.0$ & $12370 \pm 80$ \\
$5 \% \mathrm{Au}$ & $11.7 \pm 1.9$ & $11820 \pm 60$ \\
$10 \% \mathrm{Au}$ & $9.2 \pm 2.3$ & $10580 \pm 90$ \\
$15 \% \mathrm{Au}$ & $6.8 \pm 1.6$ & $8660 \pm 90$ \\
$20 \% \mathrm{Au}$ & $6.4 \pm 3.0$ & $7950 \pm 190$ \\
$40 \% \mathrm{Au}$ & $3.5 \pm 1.9$ & $8780 \pm 100$ \\
\hline \multicolumn{3}{|c|}{} \\
\hline $\mathrm{Pd}-44$ & Sputtered \\
$\mathrm{Pd}-46$ & $16.4 \pm 2.6$ & $12770 \pm 60$ \\
$\mathrm{Pd}-126$ & $18.5 \pm 0.6$ & $13400 \pm 10$ \\
& $6.4 \pm 1.2$ & $8990 \pm 70$ \\
\hline
\end{tabular}

The thinner sputtered membranes generally had higher activation energies and pre-exponential factors than their cold-worked counterparts of similar composition, producing markedly superior permeability at high temperature. The thicker membrane SwRI-126, however, had both a low activation energy and low pre-exponential factor, resulting in behavior similar to the cold-worked materials. Additionally, the two types of membranes responded differently to oxidative pre-conditioning. Previous research in sputtered Pd-Ag alloys has demonstrated that the history of a given membrane has a significant effect upon its performance, with pretreatment under air at $573 \mathrm{~K}$ (as was done in this work) producing improved hydrogen permeability [16]. Control experiments, in which membranes were heated under nitrogen rather than air, did duplicate these results for the sputtered membranes. A sample of SwRI Pd-46 pretreated in nitrogen had 92\% of the hydrogen permeability of a pre-oxidized material. Conversely, the cold-worked TKK 10\% Au did not show any permeability depression when pretreated under $\mathrm{N}_{2}$, or any improvement in permeability when aggressively pre-oxidized at 723 $\mathrm{K}$ for five hours. Potential causes for these variations in behavior are discussed in the following section.

\section{Structural and compositional analysis}

X-ray diffraction analysis was performed on all membranes before and after testing. Crystallite size can be estimated from peak breadth data using Scherrer's formula, and then averaged by peak intensity. As received, this gives a mean grain diameter for the sputtered membranes of $445 \AA$, and for the cold-worked membranes of $426 \AA$. These two values are too close to consider grain size as a determining factor in hydrogen permeability. This grain size is slightly larger than expected for a sputtered palladium film, but may be attributed to heating of the substrate, which is known to increase grain size $[17,18]$.

A selection of XRD spectra are presented in Figure 6. Both fabrication techniques produce membranes whose crystallites have a strong preferential orientation. While some preferential orientation is predicted for all non-powdered samples, this is much more significant than that observed in electrolessly plated membranes [10]. A powdered sample with completely randomly oriented grains would generally have its 111 peak (around $2 \theta=38-40$ ) be the largest peak, with the 200 phase peak (around $2 \theta=46-47$ ) having between 40 and $50 \%$ of the 111 peak's intensity. For the cold-worked membranes, the trend was reversed, with the 200 peak being much larger. This is a welldocumented condition known to occurin cold-working of sheets and foils, and is caused by the tendency of the grains to rotate during plastic deformation, and to be influenced both by the external forces applied and by the motion of adjoining grains [19]. However, preferential orientation is known to effect properties diverse as magnetism and strength, and its influence on diffusivity and solubility is not presently quantified for palladium. The SwRI 


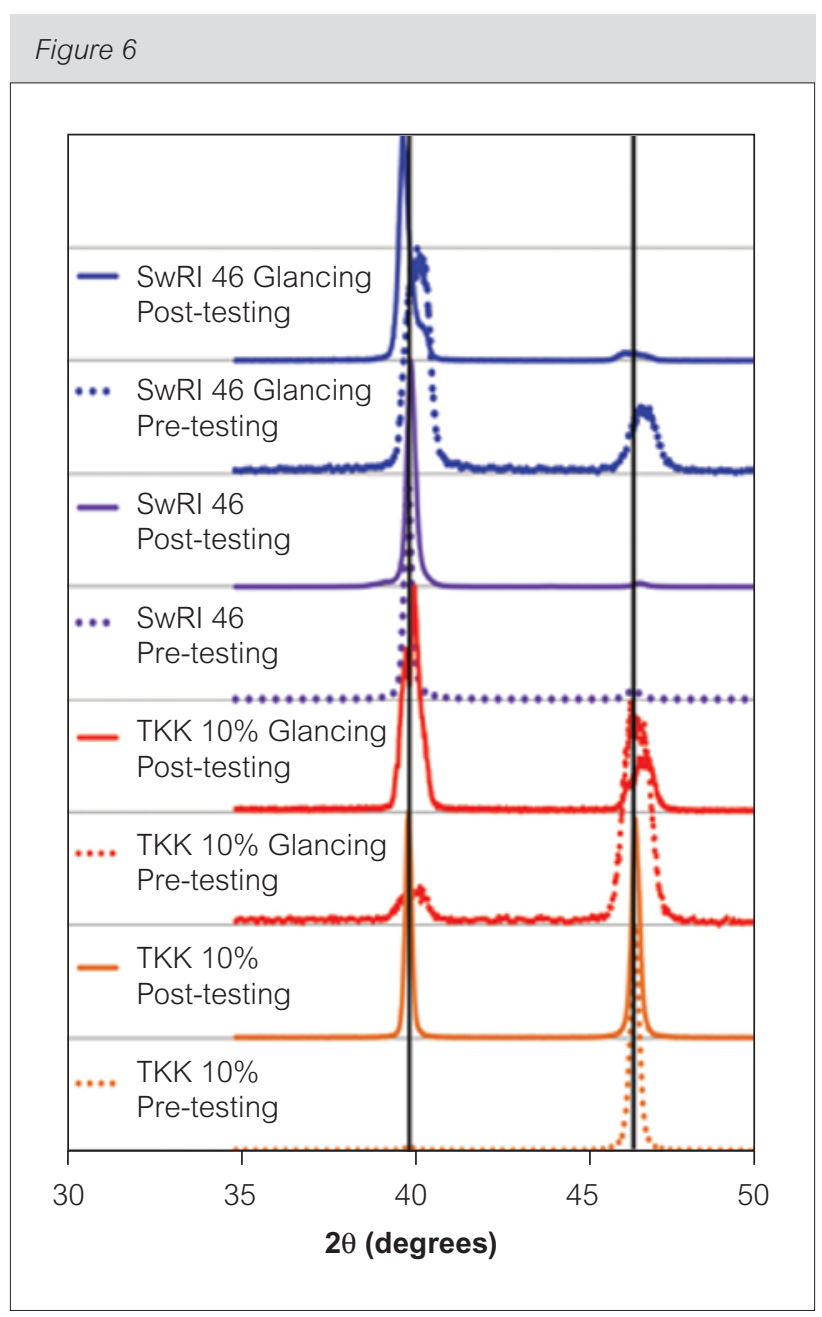

$X$-ray spectra of the feed sides of sputtered and cold-worked membranes with 9-10\% gold by weight. Predicted peak positions from Vegard's law are denoted by vertical black lines

sputtered foils had similar orientation effects, with the exception that they favored the 111 phase. This is a known phenomenon in the palladium silver system for sputtered films produced on Si-100 substrates $[20,21]$, and is promoted when the substrate is heated during deposition [22].

Peak position in the Pd-Au system can be predicted by Vegard's law, which states that there is a linear relationship to the lattice parameter in a solid solution and the concentration of constituent elements [23]. However, in both cold-worked and sputtered membranes, there is significant peak shift from the predicted values. Such behavior is typically caused by residual stress in the foil causing the lattice to be slightly distorted. The most substantial peak shift was in the $20 \mathrm{wt} \%$ Au cold-worked alloy, which also had permeability higher than predicted throughout the range of temperatures tested.
During the membrane testing process, several phenomena were observed. Predictably, exposure to high temperatures caused grain growth. This growth was most pronounced for the sputtered membranes, reaching a mean size of $643 \AA$, while cold-worked membranes reached a mean size of $520 \AA$. The literature on the effect of grain size is contradictory, with some researchers suggesting increased size has a beneficial effect on permeability and others finding the opposite [24, 25]. In this case, since low-temperature permeability showed no hysteresis before and after high-temperature exposure, it seems likely that grain size is not the determining factor in performance, although additional research is needed to determine the relative contributions of inter- and intra-granular transport.

One more obvious phenomena observed upon testing was the fluctuation of relative peak intensities. In the cold-worked membranes, the 111 relative peak intensity increased during testing, showing that the grains in the membrane were rearranging to a more random orientation. A similar result was noticed, though much more subtle, for the 200 peaks in the sputtered foils, suggesting that they are less mobile under standard test conditions. Additionally, the existing peaks shifted position under test conditions. Of the ten cold-worked $\mathrm{Pd}-\mathrm{Au} 200$ reflections, nine shifted to lower $2 \theta$, indicating a larger lattice parameter, which, according to Vegard's law, indicates a higher gold content. This may represent a segregation of gold to the surface during permeation testing. The cold-worked pure palladium membrane also underwent a peak shift, but it shifted to a higher $2 \theta$, or lower lattice parameter. The sputtered foils exhibited reflections generally shifted to a higher $2 \theta$, possibly indicating gold depletion in the bulk phase. However, the shifts were slight in comparison to those observed in the cold-worked membranes, and are probably due to relaxation of the membrane lattice with time at temperature. Theory predicts a surface segregation to the top few outer layers, but XRD is a bulk technique, and the measured effect is greater than a simple surface rearrangement [26].

No evidence of long-range ordering was found in the standard diffractograms, but glancing-incidence XRD reveals that a slightly different process took place in the top 0.6-0.8 microns of the membranes. In this near-surface region of cold-worked membranes, there is a proportionally larger 111 reflection than in the bulk of the material before permeation testing. On the feed sides, after testing, this reflection begins to split into a doublet, forming a lower- $2 \theta$ 
point. The most common reason for peak splitting is the formation of a superlattice. In a normal solid solution, such as is typical of the Pd-Au system, the solute atoms are randomly placed. In an ordered solid solution with a superlattice, the solute atoms have a periodic arrangement despite maintaining the overall structure. From the Pd-Au phase diagram, this is not likely for any of the membranes unless they undergo extreme gold segregation to the surface, producing a 60-69 wt\% Au surface alloy [27]. As will be discussed below for the EDS analysis, there is no evidence of such extensive segregation, and this doubling is probably due to the formation of a hydrogen swollen superlattice. Similar results are observed in the sputtered membranes, although the doublet peak intensity relative to the initial $\mathrm{Pd}-\mathrm{Au}$ peak is significantly greater than in the cold-worked membranes, and it occurs on both sides of the film. This implies that the sputtered membranes are taking more hydrogen into their lattices, which may account for the difference in hydrogen permeability, and may have promoted the failure of SwRI Pd-126.

Membrane analysis by EDS generally shows a slight gold enrichment of the near-surface region for all cold worked membranes, and a slight gold depletion for sputtered membranes during permeation testing, consistent with the results obtained by XRD (Table 3). The change in compositions was much less pronounced for the sputtered films, suggesting that gold was less mobile in them, perhaps due to their more stable orientation. SEM examination of the membrane before and after testing shows that both types of membrane underwent substantial changes on the sub-micron scale during testing. Cross-sectional micrographs
(Figure 7) show that both materials had initially a fine structure, with some distortion near the surface in the case of the cold-worked materials. After testing, however, recrystallization into subgrains occurred in both types of film. While this is to be expected in materials that have been shown to undergo grain growth, it suggests that future research in this area could be benefited by an annealing pretreatment before membranes are mounted in test housings. By encouraging recrystallization and reducing material stresses before constraining membrane motion by mounting it in the housing, improved stability may be produced, helping to prevent the low- $T$ failures observed in membrane SWRI-126.

XPS of cold-worked membranes prior to testing shows a slight segregation of gold to the surface, most pronounced for membranes with low gold content. After hydrogen permeation testing, both sides of the membrane generally became slightly gold-depleted (Figure 8). At gold contents of 20 wt\% or below, the feed side was preferentially depleted of gold, but this effect was reversed for the 40 wt\% Au alloy, suggesting that permeation-driven segregation as observed in the Pd-Ag system does not necessarily occur [28]. However, the trend of gold depletion from the initial composition is consistent across both surfaces. This is in direct opposition to theoretical predictions, which state that the top three surface layers will become gold-enriched upon heating to $800 \mathrm{~K}$, with the effect being more pronounced for membranes with higher gold [26]. From this, it becomes clear that although permeation-driven segregation does not occur, the surface composition is affected by the presence of high-temperature hydrogen, and

Table 3: Membrane compositions by EDS before and after testing

\begin{tabular}{|c|c|c|c|c|c|c|}
\hline & \multicolumn{4}{|c|}{$\mathbf{W t} \% \mathrm{Au}$} & \multicolumn{2}{|c|}{$\%$ Au change } \\
\hline & \multicolumn{2}{|r|}{ Feed } & \multicolumn{2}{|r|}{ Permeate } & & \\
\hline & Pretesting & Post testing & Pretesting & Post testing & Feed & Permeate \\
\hline Alfa Pd & $0 \%$ & $0 \%$ & $0 \%$ & $0 \%$ & $0 \%$ & $0 \%$ \\
\hline TKK 5\% & $5 \%$ & $7 \%$ & $5 \%$ & $9 \%$ & $2 \%$ & $4 \%$ \\
\hline TKK 10\% & $11 \%$ & $12 \%$ & $11 \%$ & $12 \%$ & $1 \%$ & $0 \%$ \\
\hline TKK 15\% & $13 \%$ & $20 \%$ & $15 \%$ & $21 \%$ & $7 \%$ & $6 \%$ \\
\hline TKK 20\% & $21 \%$ & $26 \%$ & $21 \%$ & $23 \%$ & $5 \%$ & $2 \%$ \\
\hline TKK 40\% & $38 \%$ & $43 \%$ & $41 \%$ & $46 \%$ & $5 \%$ & $5 \%$ \\
\hline SWRI Pd-44 & $9 \%$ & $7 \%$ & $7 \%$ & $7 \%$ & $-2 \%$ & $0 \%$ \\
\hline SWRI Pd-46 & $8 \%$ & $7 \%$ & $10 \%$ & $10 \%$ & $-1 \%$ & $0 \%$ \\
\hline SWRI Pd-126 & $13 \%$ & $14 \%$ & $9 \%$ & $9 \%$ & $1 \%$ & $0 \%$ \\
\hline
\end{tabular}




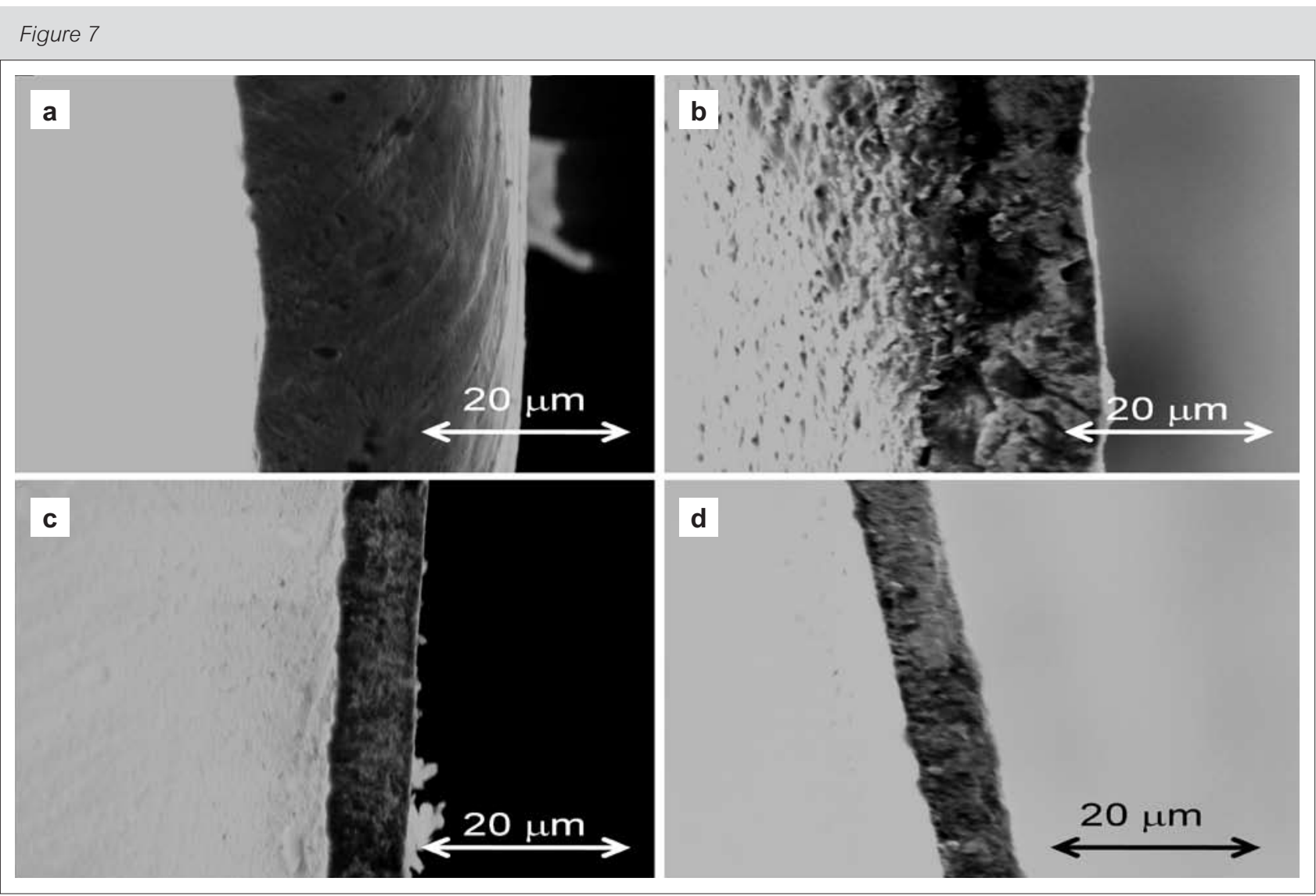

2000x cross-sectional SEM images for fractures of a) TKK 10 before testing; b) TKK 10 after testing; c)SWRI 46 before testing; d) SWRI 46 after testing

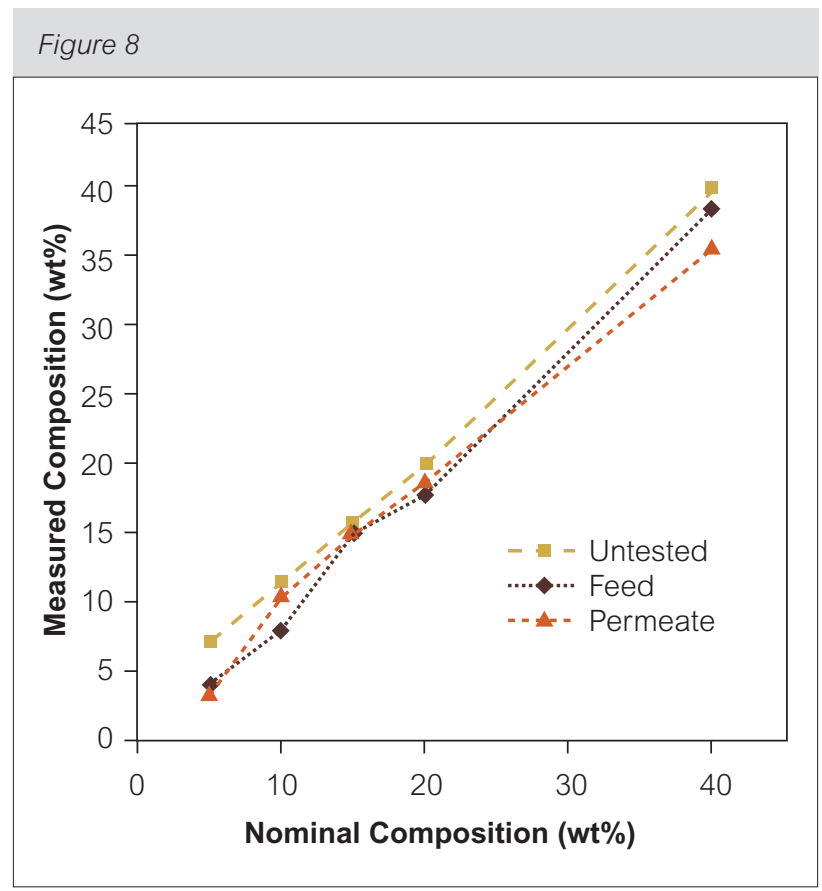

XPS measured alloy compositions as a function of nominal bulk composition for cold-worked Pd-Au membranes may also be impacted by other components in the gas stream.

When selecting a membrane material for a hypothetical hydrogen purification unit, all of these results may have an impact on the choice of material and fabrication technique. The permeability of sputtered materials being, on average, superior to the permeability of cold-worked materials makes them more initially appealing candidates. These materials also can readily be made thinner than is convenient for cold-worked materials, and can be applied to porous substrates to increase mechanical strength. This must be balanced, however, with the possibility of low-temperature failures, and the fact that performance in streams containing sulfur and carbon may also be affected by fabrication technique. A preconditioning step, such as annealing or ion bombardment, may be indicated to relieve stresses prior to utilization as a hydrogen membrane. 


\section{Conclusions}

Self-supported sputtered and cold-worked palladium and palladium-gold alloys with up to 40 wt $\%$ Au were studied under single-gas hydrogen and nitrogen permeation test conditions, and were found to be able to selectively permeate hydrogen at temperatures between 473 and $773 \mathrm{~K}$ and gas partial pressures as great as $772 \mathrm{kPa}$. No single alloy had superior permeability across the range of temperatures. The addition of up to $20 \mathrm{wt} \%$ gold acted to decrease activation energy but increase the pre-exponential factor of hydrogen permeability. Fabrication technique also played a role in membrane permeability, with cold-worked alloys generally having lower hydrogen permeability than sputtered materials of equivalent compositions.

The differences in gas permeation properties for different fabrication methods cannot be attributed to grain boundary volume increasing membrane hydrogen solubility, as grain sizes measured by XRD are the same for both films. However, the two material types have different crystallite orientations. The 200-phase dominated cold-worked membranes have a less stable orientation which promotes gold migration to the membrane surface, while the 111-phase dominated sputtered membranes do not undergo equivalent material shifts, and tend to form long-range ordered surface phases. The shifting of the metal and formation of new phases does not appear to cause any changes in hydrogen permeability, as membranes do not demonstrate hysteresis upon temperature cycling.

\section{Acknowledgements}

The authors gratefully acknowledge financial assistance from the World Gold Council through G.R.O.W. Project RP 03-08. Additional financial support to J. Douglas Way and Sabina K. Gade was provided by the U.S. Department of Energy through contracts DE-FC26-07NT-43056, DE-FC26-07NT43054, and from Grant \#DE-FG36-05G015093 from the DOE Office of Science, Basic Energy Sciences, Chemical Sciences, Geosciences, and Biosciences Division. Kent E. Coulter was supported by DOE contract DE-FC26-07NT-43054. For XPS experimentation, we thank Sarah Morgan and Dr. Timothy Ohno.

\section{About the authors}

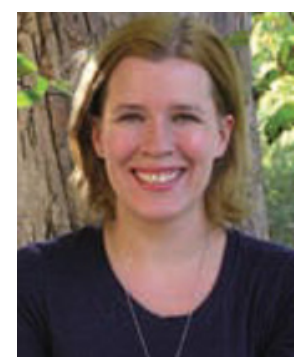

Sabina K. Gade received her B.S. from the University of Colorado at Boulder, and Ph.D from the Colorado School of Mines, both in chemical engineering. Her thesis research dealt with the development, characterization, and optimization of palladium-gold alloys for hydrogen separations. She is currently a postdoctoral researcher.

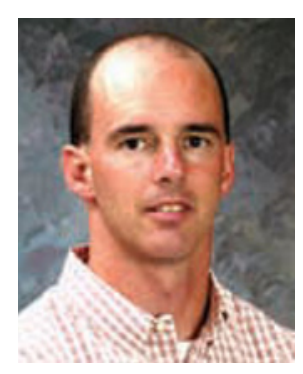

Kent E. Coulter is the Manager of Surface Engineering \& Materials Chemistry at Southwest Research Institute where he has been for 5 years. Prior to Southwest, he was a Director of Science \& Technology at JDS Uniphase. Kent earned his PhD in Physical Chemistry from Texas A\&M University and his BS in Chemistry and Mathematics from Ouachita Baptist University.

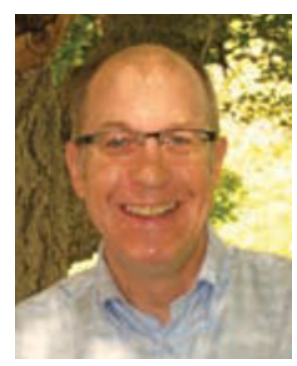

J. Douglas Way is a Professor of Chemical Engineering at the Colorado School of Mines where he has been a faculty member since 1993. Professor Way received his chemical engineering education at the University of Colorado at Boulder

\section{References}

1 X. Cheng, Z. Shi, N. Glass, L. Zhang, J. Zhang, D. Song, Z.S. Liu, H. Wang, J. Shen, Journal of Power Sources, 2007, 165,739

2 G.Q. Lu, J.C. Diniz da Costa, M. Duke, S. Giessler, R. Socolow, R.H. Williams, T. Kreutz, Journal of Colloid and Interface Science, 2007, 314, 589

3 M. Klett, J. White, R. Schoff, T. Buchanan, Hydrogen Production Facilities Plant Performance and Cost Comparisons, Parsons Infrastructure and Technology Group Inc., 2002

4 D.S. Scholl, Y.H. Ma, MRS Bulletin, 2006, 31, 770

5 S. Paglieri, J.D. Way, Separation and Purification Methods, 2002, 31, 1

6 A.W. Erickson, R.M. Paczewski, Hydrogen Purification Apparatus, U.S. Pat. No. 6,866,698 B2, 2003

7 D. McKinley, Metal Alloy for Hydrogen Separation and Purification, U.S. Pat. No. 3,350,845, 1967 
8 D.L. McKinley, Method for Hydrogen Separation and Purification, U.S. Pat. No. 3,439,474, 1969

9 D. Fort, J.P.G. Farr, I.R. Harris, Journal of the Less Common Metals, 1975, 39, 293

10 S.K. Gade, P.M. Thoen, J.D. Way, Journal of Membrane Science, 2008, 316, 112

11 O. Hatlevik, S.K. Gade, M.K. Keeling, P.M. Thoen, J.D. Way, Separation and Purification Technology, 2010, 73, 59

12 S.K. Gade, E.A. Payzant, H.J. Park, P.M. Thoen, J.D. Way, Journal of Membrane Science, 2009, 340, 22

13 T.L. Ward, T. Dao, Journal of Membrane Science, 1999, 153, 211

14 H. Li, H. Xu, W. Li, Journal of Membrane Science, 2008, 324, 44

15 I.B. Elkina, J.H. Meldon, Desalination, 2002, 147, 445

16 A.L. Mejdell, H. Klett, A. Ramachandran, A. Borg, R. Bredesen, Journal of Membrane Science, 2008, 307, 96

17 K. Bryden, J. Ying, Journal of Membrane Science, 2002, 203, 29

18 K.J. Bryden, J.Y. Ying, Materials Science and Engineering, 1995, A204, 140
19 B. Cullity, S.R. Stock, Elements of X-Ray Diffraction, Third Edition, Prentice-Hall, 2001

20 S. Tosti, L. Bettinali, S. Castelli, F. Sarto, S. Scaglione, V. Violante, Journal of Membrane Science, 2002, 196, 241

21 H.D. Tong, A.H.J. vanden Berg, J.G.E. Gardeniers, H.V. Jansen, F.C. Gielens, M.C. Elwenspoek, Thin Solid Films, 2005, 479, 89

22 R. Anton, Thin Solid Films, 1984, 118, 293

23 F. Maeland, T. Flanagan, Canadian Journal of Physics, 1964, 42, 2364

24 K. Bryden, J. Ying, NanoStructured Materials, 1997, 9, 485

25 B.A. McCool, Y.S. Lin, Journal of Materials Science, 2001, 36, 3221

26 J. Rousset, J. Bertolini, P. Miegge, Physical Review B, 1996, 53, 4947

27 T.B. Massalski, H. Okamoto, P.R. Subramian, L. Kacprzak, eds. Binary Alloy Phase Diagrams. 2nd ed. Vol. 3. 1990, ASM International

28 J. Shu, B.E.W. Bongondo, B.P.A. Grandjean, A. Adnot, S. Kaliaguine, Surface Science, 1993, 291 